\title{
Tobacco Smoke and Alcohol Consumption in Relation to Oral Cancer in Albania
}

\author{
Canga Mimoza ${ }^{1,}$, Malagnino Antonio Vito ${ }^{2}$ \\ ${ }^{1}$ Department of Public Health, University “Ismail Qemali”, Vlore, Albania \\ ${ }^{2}$ Department of Endodontics, University “Gabriele D'Annunzio”, Chieti, Italy
}

Email address:

mimoza-canga@hotmail.com (C. Mimoza)

${ }^{*}$ Corresponding author

To cite this article:

Canga Mimoza, Malagnino Antonio Vito. Tobacco Smoke and Alcohol Consumption in Relation to Oral Cancer in Albania. International Journal of Clinical Oral and Maxillofacial Surgery. Vol. 4, No. 1, 2018, pp. 30-34. doi: 10.11648/j.ijcoms.20180401.16

Received: July 3, 2018; Accepted: July 17, 2018; Published: August 31, 2018

\begin{abstract}
Objective: The purpose of this study is to investigate the relationship between tobacco, alcohol and other viruses in increasing the risk of being affected by oral carcinomas. Material and method: In our study the sample was composed by 200 patients, all Albanians. The experiment was conducted in the time period 2014-2018, in Albania. The participants were of the age range 45-75 years old and their average age was 60 years old. The ratio between males and females was 3:1, so 152 of the participants were males and 48 were females. The participants were all tobacco users, but they also had other characteristics such as: being virus carriers. More specifically, 44 patients were HPV carriers, 12 patients were EBV carriers, 16 patients were HIV carriers, 72 patients were alcohol addicted, while the remained 56 patients had no viruses they were only tobacco and marijuana smokers. Results: There were 200 patients who were smokers, so smoking is not excluded in any patient. Smoking was the dominant characteristic among participants, $36 \%$ of the patients were smokers and consumed alcohol, which increased the risk for oral carcinomas of both kinds. Based on our findings we claim that $36 \%$ of the patients smoked and drank alcohol at the same time, $36 \%$ were smokers and virus carriers, while $28 \%$ were only smokers. Conclusion: Tobacco smoking causes oral carcinomas in those patients who carry viruses, such as: HPV, HIV and EBV, although they are not regular tobacco users.
\end{abstract}

Keywords: Alcohol, Oral Carcinomas, Tobacco, Viruses

\section{Introduction}

Tobacco is the cause of at least $80 \%$ of oral cancers. [1] People who use tobacco in form of cigarettes are 4-12 times more risked by oral cancers. [2] Studies show that addictive nicotine element found in cigarettes and other smoking products activates neutrofiles in order to release molecules that increase the inflammation and cause damaging immunological effects.

Early diagnosis of preclinical or pre-cancer involves a simple, inexpensive examination of the oral cavity and early treatment provides a good prognosis for patients. [3] Oral carcinomas shows a complex, multistage developmental process and involves several environmental and genetic factors. Among these factors, the important role of smoking and alcohol in oral malignancy has drawn increasingly interest. [4] Oral carcinomas was defined by Ackerman in 1948 [5] as a diagnostically problematic squamous cell neoplasia involving lip, oropharyngeal, and laryngeal mucosa. Oral carcinomas are strongly associated with the chronic use of tobacco. [6] According to recent studies, human papillomavirus could have a potential role in the cancer development and progression. [7]

The aim of this study is to investigate the relationship between tobacco, alcohol and other viruses, in increasing the risk of being affected by oral carcinomas. Tobacco and alcohol are the most important contributing factors in oral cancer etiology. [8] Alcohol, particularly when associated with tobacco use, has been recognized as an important risk factor for mouth cancer for almost 50 years. [9] Together, they are associated with approximately $75 \%$ of upper aerodigestive tract cancers. However, intake of alcohol remains high in many countries. The rising incidence of oral 
cancer has prompted a revaluation of the role of alcohol. [9] An etiologic agent related with the development of oral carcinomas in oral cavity is characterized by clinical progression and genetic changes. [10]

Hong [11] demonstrated an increase of squamous cell carcinoma from $19 \%$ in the years $1987-1990$ to $47 \%$ in the years 2001-2005, in Australia. In the United States, Chaturvedi [12] showed a significant increase from $16.3 \%$ for the years $1984-1989$ to $72.7 \%$ in the years $2000-2004$. HIV infection increases susceptibility to opportunistic infections and viral promoted cancers. [13-14]

The associations between HIV and HPV infections in general, are well established. [15-18] HIV- infected patients are at increased risk for developing Hodgkin's disease. EBV causes persistent, reactivated infection of the lateral tongue in the immunocompromised populations, particularly in HIVpositive individuals. It is the cause of the majority of nonkeratinizing nasopharyngeal carcinomas, as well as, some oral squamous cell carcinomas. [19]

Yet, survival from oral cancer is among the lowest of all cancer sites, primarily because of the advanced stage in which the disease is diagnosed. Treatments for localized oral cancer yield five year survival rates as high as $86 \%$, compared with $42 \%$ for regional and $22 \%$ for distant disease. Only $35 \%$ of patients in the US are diagnosed at the localized stage of oral cancer. [20] Strategies that seek to increase accessibility to the dentist by minimizing financial barriers may resolve only partially the low usage of dental services by tobacco users. [21]

\section{Material and Method}

Our study was conducted in Albania in the years 20142018. The sample was composed by 200 patients, where 152 of them were males and 48 were females. Their age range was from 45-75 years old, so their average age was 60 years old. All the participants were tobacco users, but some of them were also virus carriers. More specifically, 44 patients were HPV carriers, 12 patients were EBV carriers, 16 patients were HIV carriers, 72 patients were addicted to alcohol, while the remained 56 patients had no viruses, they were only tobacco and marijuana smokers. Based on the examinations of our patients' clinical records we saw that 136 patients smoke cigarettes during the first 5 minutes after they are waken up. From our findings we saw that 128 patients used to smoke and drink alcohol, frequently, while 72 patients used to smoke and drink alcohol, rarely. So, we came at the conclusion that 160 patients are affected by squamous cell carcinoma and 40 patients are affected by verrucous cell carcinoma. This means that squamous cell carcinoma is more dominant that verrucous cell carcinoma. Patients always complained of oral and lips' continuous pain, hemorrhage and abnormal numbness inside of the mouth, edema of the jaw, difficulties while speaking and breathing problems. They were diagnosed through incision diagnostic biopsy and ortho-panoramic $\mathrm{x}$ ray. The diagnosis was confirmed with oral CT images, in order to determine the size of the lesion, its width and the involvement of the close structures to the lesion. Statistical analysis was performed using IBM SPSS Statistics 23.0. Data were analyzed by onesample t-tests. The significance level $(\alpha)$ was set at 0.05 , with a confidence interval (CI) of $95 \%$.

\section{Results}

There were 200 patients who were smokers, so smoking is not excluded in any patient. Our findings show that $68 \%$ of our sample smoke during the first 5 minutes, after they are waken up and they have the tendency to smoke 40-50 cigarettes per day and also we saw that there are patients who smoked the first cigarette after one hour they were waken up. "Figure 1"

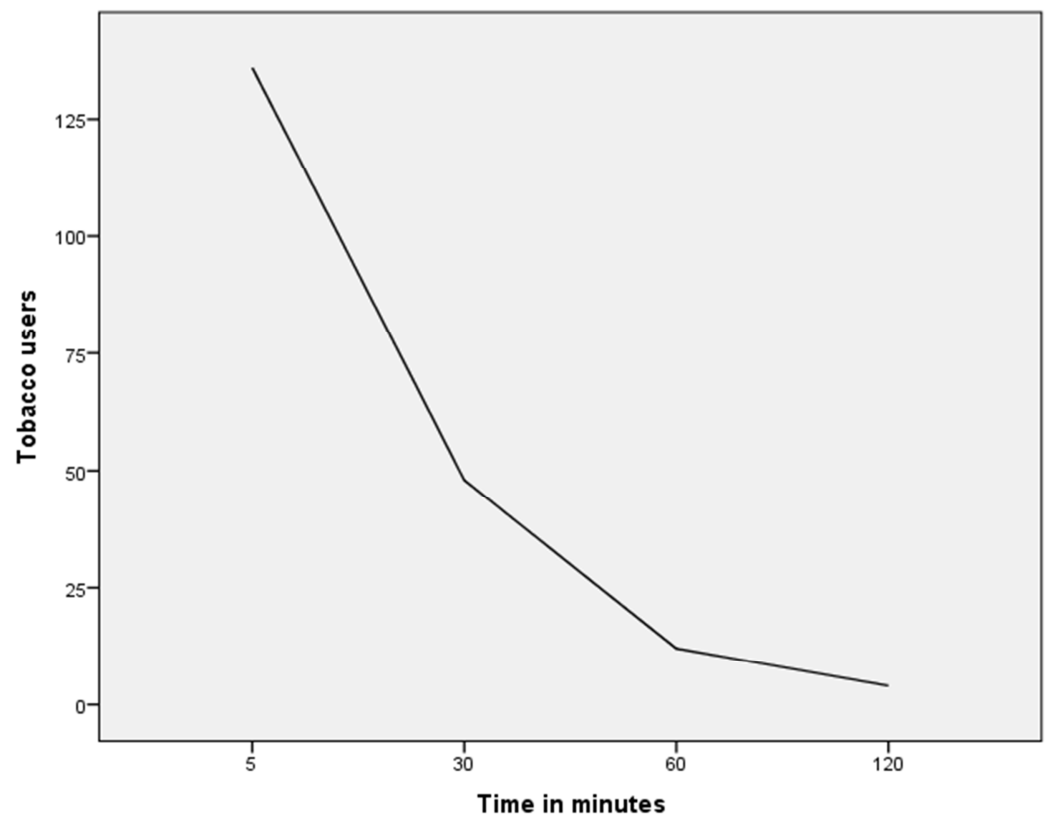

Figure 1. The presentation of the time when most of the patients smoke in the morning. 
Smoking was the dominant characteristic among participants, $36 \%$ of the patients were smokers and consumed alcohol, which increased the risk for oral carcinomas of both kinds. Based on our findings we claim that $36 \%$ of the patients smoked and drank alcohol at the same time, $36 \%$ were smokers and virus carriers, while $28 \%$ were only smokers. We also noticed that in the group of 200 patients there was the presence of squamous and verrucous carcinoma. The charts showed that these patients other than smoking and drinking were also carriers of viruses such as HPV, HIV and EBV. "Figure 2"

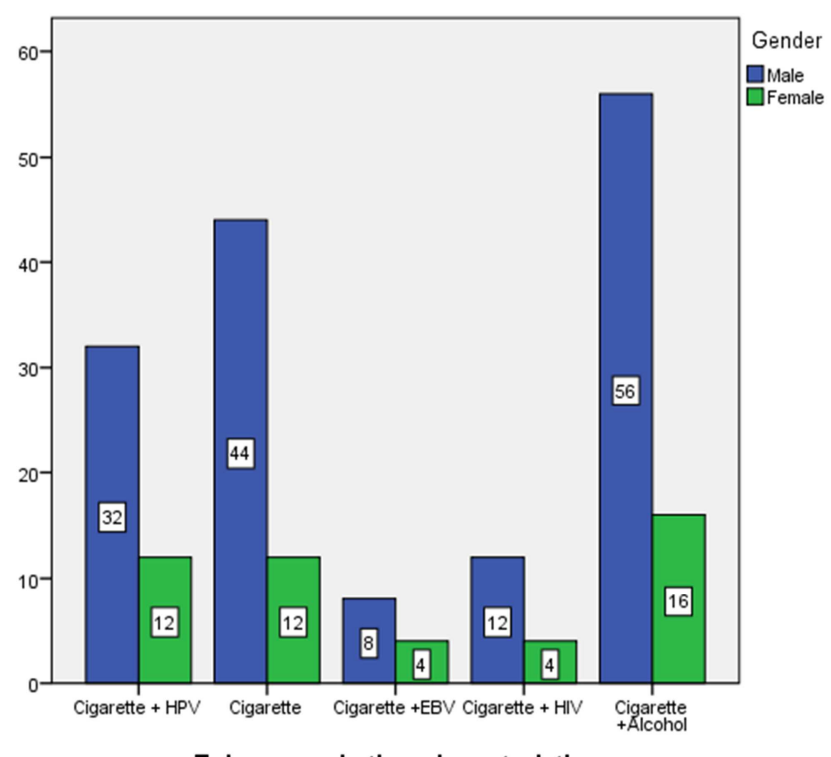

Tobacco and other characteristics

Figure 2. Shows the number of males and females, who before being diagnosed with oral carcinomas were smokers, drank alcohol and were virus carriers.

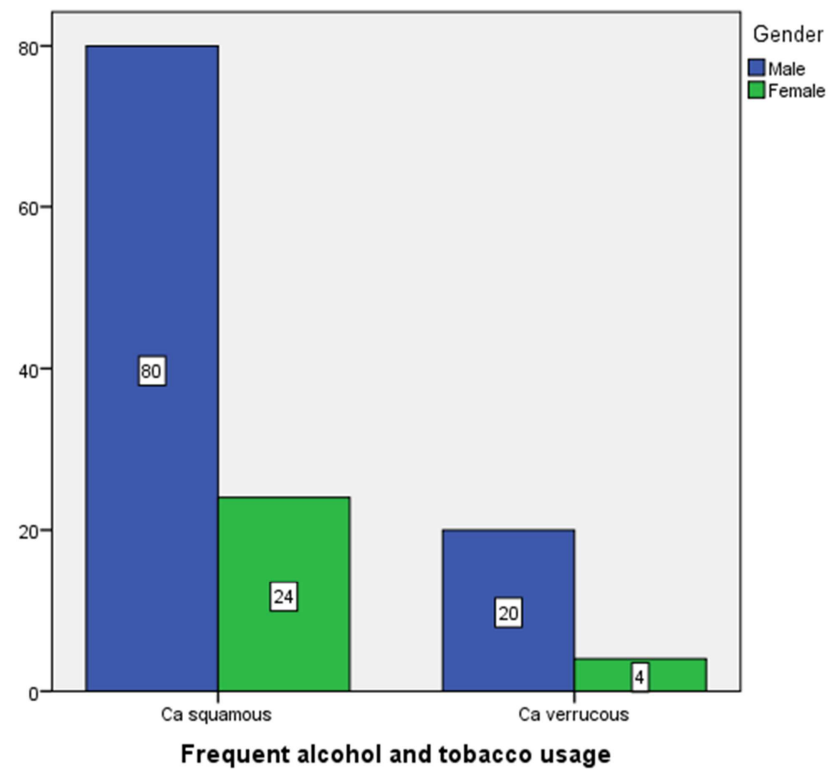

Figure 3. Shows the number of patients who are affected by oral carcinomas because of the frequent usage of alcohol and tobacco.

Smoking and drinking causes oral carcinomas in dramatic values. There were 128 patients who use tobacco and alcohol, frequently. $81.3 \%$ of them were affected with squamous cell carcinoma, while $18.7 \%$ of the patients were affected by verrucous cell carcinoma. "Figure 3"

There were also 72 patients who rarely consumed tobacco and alcohol but were carriers of HPV, HIV and EBV. They also had squamous and verrucous cell carcinomas. Based on our findings patients affected with both squamous and verrucous cell carcinomas were 52 males who dominated to female patients that were only 20 . "Figure 4"

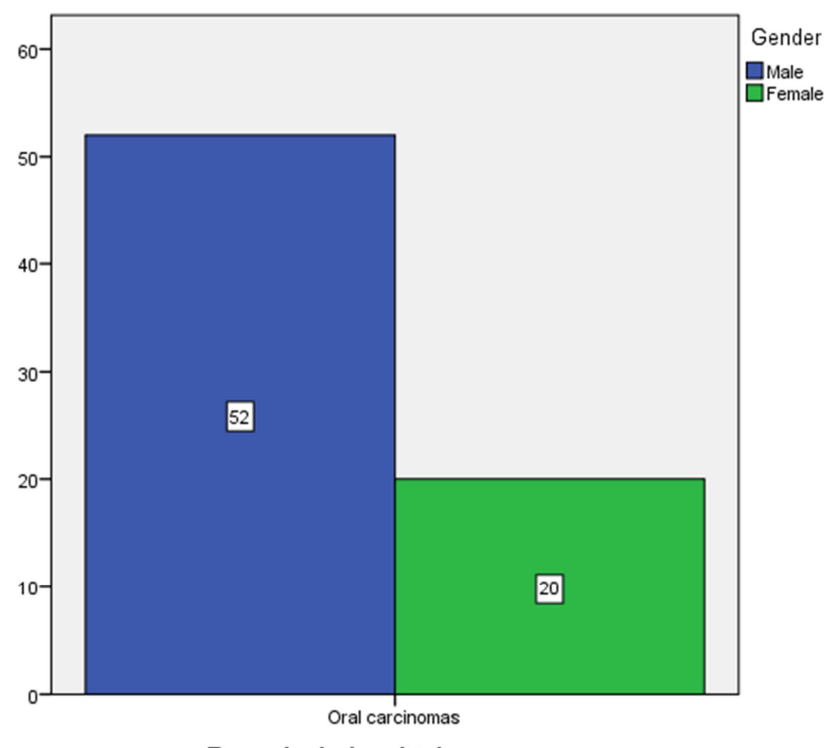

Rare alcohol and tobacco usage

Figure 4. Shows the number of patients who are affected by oral carcinomas although they rarely use alcohol and tobacco.

The data showed that there is a strong and significant relation between smoking, the abuse with alcohol and viruses, in the manifestation of squamous and verrucous cell carcinomas. [Table 1]

Table 1. Tobacco and other characteristics in relation to oral carcinomas ${ }^{l}$.

\begin{tabular}{llllll}
\hline & SD & t & Sign & CI 95\% & P-value \\
\hline $\begin{array}{l}\text { Tobacco and other } \\
\text { characteristics }\end{array}$ & 1.639 & 26.57 & .000 & $2.85-3.31$ & $P<0.05$ \\
Oral carcinomas & .401 & 42.32 & .000 & $1.14-1.26$ & $P<0.05$ \\
\hline
\end{tabular}

\section{Discussion}

In our study the ratio between males and females was $3: 1$. Based on our findings $64 \%$ of the patients use tobacco and carry viruses such as: EBV, EPV, HIV. $76 \%$ of males were virus carriers and alcohol addicts who got affected by oral cancers. While $24 \%$ of women are virus carriers and use both tobacco and alcohol. Hooper [22] in their systematic review claimed that the majority of cases of oral cancer have been related to tobacco use and heavy alcohol consumption. Their results support our findings and our conclusions. In order to

$1 \mathrm{SD}=$ Standard deviation $\mathrm{t}=\mathrm{t}$-test $\mathrm{Sign}=\mathrm{P}$ value $\mathrm{CI}=$ Confidence Interval 
decrease the incidence of being affected by oral carcinomas, we recommend that routine dental visits are a good way to prevent and to raise people's awareness and this is also proved by other previous investigations. [23-26]

According to Ramseier [27] dentists should make their patients aware about tobacco usage and for this he proposed routine smoking history check-ups using a simple questionnaire, both for new patients on their first visit and during subsequent check-ups. The questionnaire would determine whether patients have a mild (1-5 cigarettes per day), moderate (more than 10 cigarettes per day) or severe (more than 20 cigarettes per day) addiction. Our findings show that $68 \%$ of our sample smoke during the first 5 minutes, after they are waken up and they have the tendency to smoke 40-50 cigarettes per day.

In order to decrease the incidence of oral carcinomas we recommend that dentists should advice their patients about how to prevent and stop smoking, also people should be aware of alcohol risks, because tobacco smoking and alcohol consumption are harmful to human health.

\section{Conclusion}

Tobacco smoking causes oral carcinomas in those patients who carry viruses, such as: HPV, HIV and EBV, although they are not regular tobacco users.

\section{Financial Support and Sponsorship}

Nil.

\section{Conflicts of Interest}

There are no conflicts of interest.

\section{Acknowledgements}

I want to thank professor Malagnino for his support, contribution and the time he dedicated to this study.

\section{Informed Consent}

Informed consent of the patients was obtained for the conduction of this study. A copy of the written consent is available, at any time, for review by the editor of this journal.

\section{References}

[1] Douglas E. Morse, Walter J. Psoter, Deborah Cleveland, Donald Cohen, Mireseyed Mohit-Tabatabai, Diane L. Kosis, and Ellen Eisenberg. Smoking and drinking in relation to oral cancer and oral epithelial dysplasia. Cancer Causes Control. 2007 Nov; 18(9): 919-929.

[2] Abdoul Hossain Madani, Madhurima Dikshit, Debanshu Bhaduri, Teamur Aghamolaei, Seyed Hamid Moosavy, Ali Azarpaykan. Interaction of Alcohol Use and Specific Types of Smoking on the Development of Oral Cancer. Int J High Risk
Behav Addict. 2014 Mar; 3(1): e12120.

[3] Ummar Mangalath, Sachin Aslam Aslam, Abdul Hafiz Kooliyat Abdul Khadar, Pulikkan George Francis, Muhamed Shaloob Karimbil Mikacha, Jubin Hassan Kalathingal. Recent trends in prevention of oral cancer. J Int Soc Prev Community Dent. 2014 Dec; 4(Suppl 3): S131-S138.

[4] Xinhua Wang, Ji Xu, Lijuan Wang, Chao Liu, Huiming Wang. The role of cigarette smoking and alcohol consumption in the differentiation of oral squamous cell carcinoma for the males in China. 2015, 11(1): 141-145.

[5] Ackerman LV. Verrucous carcinoma of the oral cavity. Surgery 1948; 23: 670-678.

[6] Gross A. J, Lackland D. T. Oral cancer and smokeless tobacco: Literature review and meta-analysis. 1995;21(4): 381-394.

[7] Sung Hee Lee, Chang-Ryul Lee, Nicole Kristina Rigas, Reuben H. Kim, Mo K. Kang, No-Hee ParkKi-Hyuk Shin. Human papillomavirus 16 (HPV16) enhances tumor growth and cancer stemness of HPV-negative oral/oropharyngeal squamous cell carcinoma cells via miR-181 regulation. 2015; 4:116-125.

[8] Malay Kumar, Ronak Nanavati, Tapan G Modi, Chintan Dobariya. Oral cancer: Etiology and risk factors: A review. 2016; 12(2): 458-463.

[9] Ogden GR. Alcohol and oral cancer. Alcohol. 2005 Apr; 35(3):169-73.

[10] Hari Ram, Jayanta Sarkar, Hemant Kumar, Rituraj Konwar, M. L. B. Bhatt, Shadab Mohammad. Oral Cancer: Risk Factors and Molecular Pathogenesis. J Maxillofac Oral Surg. 2011 Jun; 10(2): 132-137.

[11] Hong AM, Grulich AE, Jones D. Squamous cell carcinoma of the oropharynx in Australian males induced by human papillomavirus vaccine targets. Vaccine 2010; 28:3269-72. [PubMed]

[12] Chaturvedi AK, Engels EA, Pfeiffer RM. Human papillomavirus and rising oropharyngeal cancer incidence in the United States. J Clin Oncol 2011; 29:4294-301. [PubMed]

[13] Frisch M, Biggar RJ, Engels EA, Goedert JJ. Association of cancer with AIDS-related immunosuppression in adults. JAMA 2001; 285:1736-1745.

[14] Hagensee ME, Cameron JE, Leigh JE, Clark RA. Human papillomavirus infection and disease in HIV-infected individuals. Am J Med Sci 2004; 328:57-63. [PubMed]

[15] Martel-Renoir D, Grunewald V, Touitou R. Qualitative analysis of the expression of Epstein-Barr virus lytic genes in nasopharyngeal carcinoma biopsies. J Gen Virol 1995; 76:1401-1408. [PubMed]

[16] Shimakage M, Horii K, Tempaku A. Association of EpsteinBarr virus with oral cancers. Hum Pathol 2002; 33:608-614. [PubMed]

[17] Shroyer KR, Greer RO, Fankhouser CA, McGuirt WF, Marshall R. Detection of human papillomavirus DNA in oral verrucous carcinoma by polymerase chain reaction. Modern Pathology 1993; 6: 669-672.

[18] Wong M, Pagano JS, Schiller JT. New Associations of human papillomavirus, simian virus 40, and Epstein-Barr virus with human cancer. J Natl Cancer Inst 2002; 94:1832-1836. [PubMed] 
[19] Michael S. McLemore, Missak Haigentz, Jr., Richard V. Smith, Gerard J. Nuovo, Llucia Alos, Antonio Cardesa, Margaret Brandwein-Gensler. Head and Neck Squamous Cell Carcinomas in HIV-Positive Patients: A Preliminary Investigation of Viral Associations. Head Neck Pathol. 2010 Jun; 4(2): 97-105.

[20] Mucci L. A, Brooks D. R. Lower use of dental services among long term cigarette smokers. J Epidemiol Community Health 2001; 55:389-393.

[21] Elena Borreani, Desmond Wright, Sasha Scambler, Jennifer E Gallagher. Minimising barriers to dental care in older people. BMC Oral Health. 2008; 8: 7.

[22] Hooper SJ, Wilson MJ, Crean SJ. Exploring the link between microorganisms and oral cancer: a systematic review of the literature. Head Neck 2009; 31:1228-1239. [PubMed]
[23] Moreno-López LA, Esparza-Gómez GC, González-Navarro A, Cerero-Lapiedra R, González-Hernández MJ, DomínguezRojas V. Risk of oral cancer associated with tobacco smoking, alcohol consumption and oral hygiene: a case-control study in Madrid, Spain. Oral Oncol 2000; 36:170-174. [PubMed].

[24] Rosenquist K. Risk factors in oral and oropharyngeal squamous cell carcinoma: a population-based case-control study in southern Sweden. Swed Dent J Suppl 2005; 179:166. [PubMed].

[25] Rothman KJ. Epidemiology of head and neck cancer. Laryngoscope 1978; 88:435-8.

[26] Urberuaga Erce M. Càncer oral. FMC 2012; 19: 321-330.

[27] Ramseier CA. Smoking prevention and cessation. Oral Health Prev Dent 2003; 1:427-39. [PubMed]. 